\title{
DACRIOCISTORRINOSTOMÍA TRANSCANALICULAR CON LÁSER DIODO EN EL TRATAMIENTO DE LA OBSTRUCCIÓN PRIMARIA ADQUIRIDA DEL CONDUCTO NASOLAGRIMAL
}

\section{Transcanalicular laser dacryocystorhinostomy in the treatment of primary acquired nasolacrimal infrasacal duct obstruction}

\author{
Paula M. de las HERAS-FLÓREZ1 ${ }^{1}$, Sara FERNÁNDEZ-CASCÓN², Elisa GIL-CARCEDO-SAÑUDO1, Isidora \\ RETTIG-INFANTE ${ }^{1}$, Javier HERRERO- AGUSTÍN ${ }^{1}$, David HERRERO-CALVO ${ }^{1}$ \\ ${ }^{1}$ Hospital Universitario Río Hortega de Valladolid. Servicio de Otorrinolaringología. Valladolid. España. \\ ${ }^{2}$ Complejo Asistencial Universitario de León. Servicio de Otorrinolaringología. León. España.
}

Correspondencia: pherasfl@saludcastillayleon.es

Fecha de recepción: 16 de diciembre de 2020

Fecha de aceptación: 11 de enero de 2021

Fecha de publicación: 13 de enero de 2021

Fecha de publicación del fascículo: 1 de junio de 2021

Conflicto de intereses: Los autores declaran no tener conflictos de intereses

Imágenes: Los autores declaran haber obtenido las imágenes con el permiso de los pacientes

Política de derechos y autoarchivo: se permite el autoarchivo de la versión post-print (SHERPA/RoMEO)

Licencia CC BY-NC-ND. Licencia Creative Commons Atribución-NoComercial-SinDerivar 4.0 Internacional

Universidad de Salamanca. Su comercialización está sujeta al permiso del editor

RESUMEN: Nuestro objetivo es describir como realizamos la dacriocistorrinostomía (DCR) transcanalicular con láser diodo modificada conjuntamente los servicios de ORL y Oftalmología en el Hospital Universitario Río Hortega de Valladolid como tratamiento quirúrgico de la obstrucción primaria adquirida infrasacal del ducto nasolagrimal. Esta patología es bastante frecuente en pacientes de edad media y avanzada que origina epífora y blefaritis, así como dacriocistitis crónica o recurrente. En el tratamiento de esta entidad se sigue considerando, aunque cada vez menos, la dacriocistorrinostomía (DCR) externa como el gold standard con unas tasas de éxito que se sitúan alrededor del $85 \%$. Sin embargo, en la actualidad, es considerada como una opción "invasiva”, que pone en riesgo estructuras como el mecanismo de bomba canalicular y puede generar una cicatriz poco cosmética. Estos riesgos potenciales se pueden evitar con técnicas menos invasivas (DCR endoscópica y la DCR transcanalicular con láser diodo modificada). La 


\section{DACRIOCISTORRINOSTOMÍA TRANSCANALICULAR CON LÁSER DIODO EN EL TRATAMIENTO DE LA OBSTRUCCIÓN PRIMARIA ADQUIRIDA DEL CONDUCTO NASOLAGRIMAL HERAS-FLÓREZ PM ET AL.}

principal desventaja que puede presentar la técnica quirúrgica de dacriocistorrinostomía con láser diodo es la inversión económica por el alto coste del aparataje y su mantenimiento.

PALABRAS CLAVE: Dacriocistorrinostomía, epífora, láser

SUMMARY: Our aim is to describe how we perform laser transcanalicular modified dacryocystorhinostomy (DCR) the departments of ENT and Ophthalmology in HURH in Valladolid as surgical treatment of primary acquired nasolacrimal infrasacal duct obstruction (PANDO). PANDO is a common disorder in middle aged an older patient leading to chronic epiphora and blepharitis as well as recurring or chronic dacryocystitis. In the treatment of PANDO, external dacryocystorhinostomy (DCR) is still considered to be "the gold standard" with success rates above $85 \%$. However, in recent years external DCR is considered an invasive procedure that puts at risk the medial structures of the eyelid including the physiological canalicular pump mechanism and leave pa-tient with an uncosmetic scar. These risks are potentially avoidable by performing less invasive techniques or choosing an endonasal approach (endoscopic or laser modified). One disad-vantage of laser modified surgery is the economic inversion because of the high cost of the equipment and maintenance.

KEYWORDS: Dacryocystorhinostomy; epiphora; laser

\section{INTRODUCCIÓN}

La DCR es una técnica tradicional en el tratamiento de la obstrucción primaria adquirida del ducto nasolagrimal, en la que el objetivo es el restablecimiento del flujo lagrimal normal mediante la creación de una comunicación entre el saco lagrimal y la fosa nasal.

Desde que en el año 1904 Toti describiera la DCR externa se han desarrollado varios procedimientos alternativos para el tratamiento de esta patología.

La primera referencia al uso de endoscopios en la DCR es del año 1988 y fue realizada por Rice y posteriormente por McDonogh y Meiring en 1989 [1]. En 1990 Massaro et al. introducen el láser endonasal usando un dispositivo láser de argón de alta energía con microscopio quirúrgico.

La DCR transcanalicular con láser fue realizada por primera vez en cadáveres por Levin en 1992.

El gran desarrollo de la endoscopia y la mejora de los sistemas de láser han dado como resultado que la combinación de ambos nos permita crear la comunicación entre el saco lagrimal y la fosa nasal, que es el propósito de la DCR, evitando incisiones y las consiguientes cicatrices faciales, además de preservar la bomba lagrimal, reducir el riesgo de sangrado y disminuir el tiempo quirúrgico, y con una rápida recuperación del paciente. Estas técnicas se pueden realizar en pacientes de edad avanzada y con comorbilidades aportando mayor seguridad.

Además, la gran evolución de la endoscopia en las últimas décadas ha favorecido que las tasas de éxito se hayan ido incrementando y según algunas series sean superiores a las de la DCR externa [2].

En general los objetivos se focalizan en conseguir técnicas lo más simples posible, menos agresivas que mantengan buenos resultados a largo plazo.

Las tasas de éxito oscilan entre el $80-90 \%$ en los primeros meses y se reducen al 70-75\% durante el primer año [3].

Se han descrito técnicas láser modificadas en las que se realiza escisión de mucosa y ampliación de la osteotomía con un abordaje combinado endonasal aumentando así las tasas de éxito $[3,4,5,6,7]$.

El propósito de este capítulo es describir como realizamos esta técnica en colaboración los Servicios de Oftalmología y ORL del Hospital Universitario 
Río Hortega de de Valladolid. El tratamiento de esta patología mediante esta técnica obtiene mejores resultados en la realización conjunta por los dos servicios como un equipo.

\section{TÉCNICA QUIRÚRGICA}

\section{EVALUACION PREQUIRÚRGICA \\ 2. PROCEDIMIENTO QUIRURGICO \\ 3. SEGUIMIENTO POSTOPERATORIO}

\section{EVALUACIÓN PREQUIRÚRGICA}

Todos los pacientes con obstrucción primaria adquirida del conducto lacrimonasal candidatos a intervención son evaluados por ambos servicios quirúrgicos.

En primer lugar, se realiza una exhaustiva historia clínica con antecedentes de patología oftalmológica y ORL, así como de patología general y tratamientos crónicos, fundamentalmente toma de anticoagulantes o antiagregantes, ya que en la mayoría de los casos los pacientes son de edad avanzada.

En la consulta de oftalmología se realiza un examen físico prestando especial atención a los párpados, puntos lacrimales superior e inferior $\mathrm{y}$ el drenaje de la lágrima.

Se procede posteriormente a una irrigación con cánula de vías lagrimales en los puntos superior e inferior. En el caso de obstrucción infrasacal el suero refluye por el punto contralateral sin que alcance la faringe del paciente [8].

Tras la confirmación de la obstrucción el paciente es evaluado por el servicio de ORL.

Se realiza una historia clínica enfocada en patología nasosinusal (poliposis, rinitis, dificultad respiratoria nasal, antecedentes traumáticos faciales o nasales, cirugías previas).

Se explora la fosa nasal visualizando posibles desviaciones septales que dificulten la visión de la zona del saco (margen anterior del cornete medio) u otras patologías nasosinusales como poliposis que requieran tratamiento en algunos casos previo a la intervención y en otras ocasiones durante la misma $[9,10]$.

Según algunos estudios, en el caso de alteraciones anatómicas leves o moderadas no se ha demostrado que su intervención quirúrgica mejore los resultados; sí estaría indicado realizar cirugía en alteraciones importantes, como sería el caso en desviaciones septales severas $[9,10]$.

La solicitud de TC se reserva para patología nasosinusal asociada y siempre en el caso de reintervenciones (DCR fallida o por patología nasosinusal).

En la realización del procedimiento es necesaria la firma de un consentimiento informado tras una explicación clara y concisa de la intervención.

\section{PROCEDIMIENTO QUIRÚRGICO}

En nuestro hospital realizamos la intervención quirúrgica con anestesia general y CMA (cirugía mayor ambulatoria).

Se realiza intubación orotraqueal con tubo anillado o mascarilla laríngea anillada.

Colocación de lentinas con vasoconstrictor nasal (oximetazolina) en la fosa nasal durante 5 minutos.

Tras retirar las lentinas se procede a introducir el endoscopio nasal hasta visualizar el margen anterior del cornete medio.

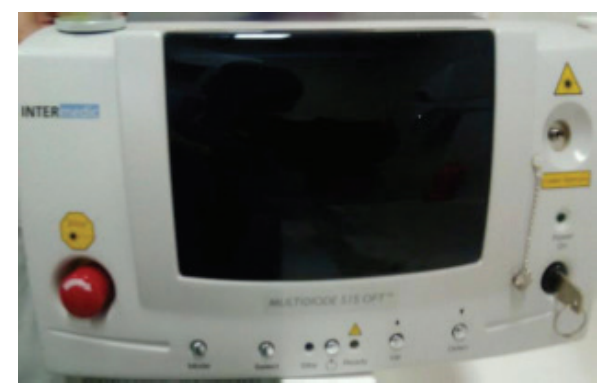

Figura 1. Equipo láser INTERMEDICS. 
En el Hospital Universitario Río Hortega utilizamos el equipo de láser Intermedics. (Figuras 1 y 2)

Se dilatan ambos puntos lagrimales (Figura 3) y se procede a introducir la fibra por el punto lagrimal superior en orientación horizontal al principio y luego vertical avanzando con delicadeza sin forzar.

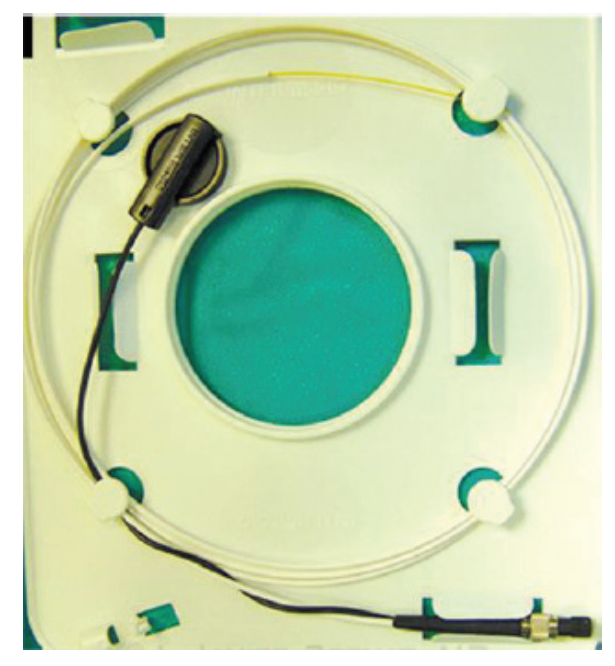

Figura 2. Sonda equipo láser

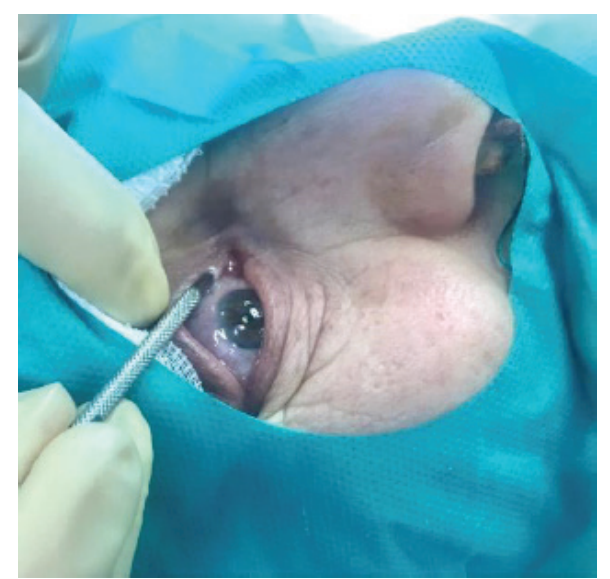

Figura 3. Dilatación punto lagrimal inferior.
Para tratar de minimizar en lo posible el daño térmico en los canalículos se introduce con la sonda de vías viscoelástico en los canalículos y el saco.

Una vez que tenemos la fibra sobre el hueso se realizan los disparos (10W), y para permitir visualizar por transiluminación la localización de la fibra de láser se disminuye la luz del endoscopio introducido en la fosa nasal. (Figuras 4 y 5 )

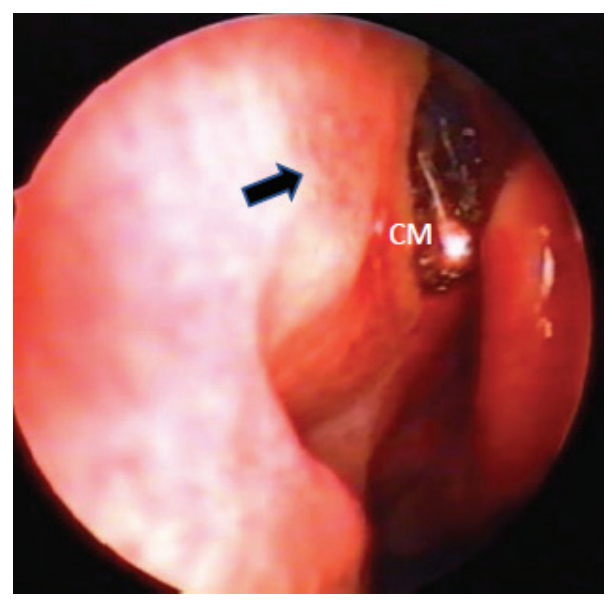

Figura 4. Sonda de láser en la apertura del saco fosa nasal derecha (flecha). Cornete medio (CM)

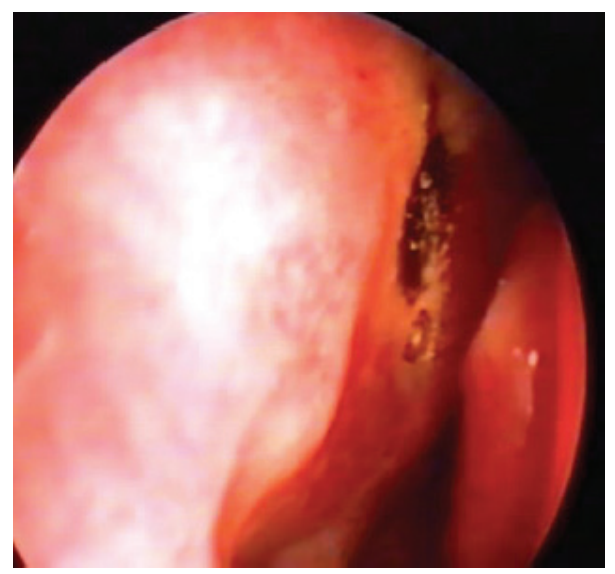

Figura 5. Imagen osteotomía láser. 
Una vez realizada la osteotomía por ambos canalículos, se amplía la misma desde la fosa nasal con pinzas de Blakesley o Kerrison [4,5].

En nuestro caso no colocamos sonda bicanalicular ni utilizamos mitomicina $\mathrm{C}$ si la cirugía no ha sido compleja (sangrado abundante, poco espacio) y si no se trata de una reintervención.

Tras la realización y ampliación de la osteotomía colocamos la sonda de vías por ambos canalículos y se realizan irrigaciones con fluoresceína para comprobar el tamaño y la permeabilidad.

Si el procedimiento ha producido erosiones en el septum o hay sospecha de que se pueda producir una sinequia colocamos placas de fluoroplástico suturada en el septum durante 15 días.

Pacientes diagnosticados de obstrucción bilateral se procede a realizar el procedimiento en ambos ojos en el mismo acto quirúrgico.

No se coloca de forma sistemática taponamiento nasal, sólo en aquellos casos en los que existe sangrado abundante durante la cirugía. Lo habitual es dejar un pequeño taponamiento con lentinas impregnadas en agua oxigenada que se retiran poco antes del alta hospitalaria.

\section{SEGUIMIENTO POSTOPERATORIO}

El paciente queda ingresado unas horas hasta su alta domiciliaria el mismo día, se trata de un procedimiento definido como CMA.

Se pauta gotas antibióticas con tobramicina oftalmológicas durante 7 días, lavados nasales y corticoterapia intranasal durante un mes.

Se revisa al paciente una semana tras la cirugía, por parte del ORL se realizan curas endoscópicas para retirada de costras (aunque no hay un criterio claro sobre su beneficio) y evaluación por oftalmología la permeabilidad de la vía mediante sondaje e irrigación $[11,12]$.

Las sondas bicanaliculares, en caso de colocación, se retiran habitualmente a los 3 meses.

Posteriormente se revisan los pacientes a los tres meses y a los seis mediante instilación de fluoresceína en el ojo y valoración endoscópica para ver permeabilidad de la vía.

\section{CAUSAS DE FRACASO DE LA TÉCNICA}

Según revisión de la literatura las causas más comunes de fallo de la técnica son:

1. Anómala localización del saco.

2. Formación de tejido de granulación alrededor de la sonda.

3. Espícula ósea.

4. Inadecuada extirpación de la pared medial del saco.

5. Sinequia entre la pared lateral nasal y el cornete medio.

Una osteotomía demasiado caudal es también una causa importante (en algunas series hasta del $28.3 \%$ ) de fracaso. Esto se produce frecuentemente porque el hueso en la parte inferior es más fino que en la parte superior (proceso frontal del maxilar) y debe de ser extirpado con fresa o pinza de Kerrison por lo que en muchas ocasiones solamente se accede al saco por la parte inferior sobre todo en la DCR láser.

Otras causas de fallo son la canaliculitis asociada (muchas ocasiones por daño térmico por la sonda láser que tratamos de minimizar con gel viscolástico), laxitud de los párpados y canaliculitis preexistente sin relación con daño térmico.

Pese a los buenos resultados obtenidos en general con esta técnica, también se ha comprobado la influencia del tiempo en las tasas de éxito [13]. Se ha visto que la obstrucción del sistema lacrimal puede disminuir el éxito durante los primeros cinco años. Los resultados de otras series han encontrado una disminución significativa en el primer año con una disminución gradual pero constante con el tiempo [2]. Esto puede ser explicado porque el riesgo de estenosis decrece con el tiempo [13] y es probablemente mayor en el primer año después de la intervención quirúrgica $[13,14]$. 


\section{CONCLUSIONES}

La DCR transcanalicular con láser diodo ampliada es un procedimiento rápido, sencillo y mínimamente invasivo en el tratamiento de la epífora por obstrucción primaria adquirida del conducto nasolagrimal muy indicada en pacientes de edad avanzada con comorbilidades.

La DCR transcanalicular con láser diodo aporta ventajas sobre todo en lo que se refiere al respeto del mecanismo fisiopatológico de la lágrima, cosméticas y de pronta recuperación.

Se debe realizar una valoración exhaustiva por ambos servicios implicados (oftalmología y ORL) previa a la intervención, realizando un correcto diagnóstico de la obstrucción y una evaluación ORL que nos permita valorar la fosa nasal con posible patología coexistente (desviaciones septales, poliposis, rinitis).

El TC se reserva para patología nasal asociada y reintervenciones por fallo en procedimientos previos como por cirugía nasal previa.

Las tasas de éxito, aunque algo menores que en otros procedimientos (DCR endoscópica o DCR externa) son bastante altas y se incrementan realizando técnicas modificadas como puede ser el aumento endonasal de la osteotomía realizada por el láser y la escisión endonasal de mucosa.

El uso de mitomicina $\mathrm{C}$ y de sondaje bicanalicular lo reservamos para reintervenciones o casos primarios complicados (introducción traumática de la sonda, sangrado profuso).

El uso de la luz del láser diodo lo utilizamos en el caso de reintervenciones (DCR fallidas o intervenciones nasales previas (poliposis, tumores...) como referencia para la localización del saco sobe todo en aquellos de situación anómala o pequeño tamaño si existen dudas en el acto quirúrgico.

\section{AGRADECIMIENTOS}

Mi agradecimiento al Dr. Guerra Linares y a la Dra. Francia que me iniciaron en el camino de esta colaboración entre ORL y oftalmología tan fructífera en mi carrera profesional.

\section{BIBLIOGRAFÍA}

1. McDonongh M; Meiring H. Endoscopic Transnasal Dacryocystorhinostomy. J Laryngol Otol 1989; 103: 585-7.

2. Cohen O, Amos I, Halperin D, Bavnik Y, Milstein A, Shoshani Y, Leiba H, Warman M. Fiveand 10-Year Outcomes for Primary Endoscopic Dacryocystorhinostomy: Failure Rate and Risk Factors. Laryngoscope. 2021 Jan;131(1):10-6..

3. Damous Feijó ED, Alves Caixeta JA, de Souza Nery AC, Murillo Limongi RM, Matayoshi S. A Comparative Study of Modified Transcanalicular Diode Laser Dacryocystorhinostomy versus Conventional Transcanalicular Diode Laser Dacryocystorhinostomy. Eur Arch Otorhinolaryngol. 2017;274:3129-34.

4. Goel R, Nagpal S, Kumar S, Meher R, Kamal S, Garg S. Transcanalicular Laser Assisted Dacryocystorhinostomy with Nasal Augmentation in Primary Nasolacrimal Duct Obstruction. Ophthalmic Plast Reconstr Surg 2017; 33(6): 408-12.

5. Maeso Riera J, Sellarés Fabrés MT. Dacriocistorrinostomía Transcanalicular con Láser Diodo: Variaciones Técnicas y Resultados. Acta Otorrinolaringol Esp. 2007;58(1):10-5.

6. Nacaroglu SA, Ogreden S, Yilmaz A, Atalay K, Kirgiz A. Comparison of Outcomes of Conventional Transcanalicular Laser Dacryocystorhinostomy and Modified Transcanalicular Laser Dacryocystorhinostomy. Wideochir Inne Tech Maloinwazyjne.2018 Sep; 13(3):401-6.

7. Mor JM, Matthaei M, Schrumpf H, Koch KR, Bölke E, Heindl LM. Transcanalicular Laser Dacryocystorhinostomy for Acquired Nasolacrimal Duct Obstruction: An Audit of 104 Patients. Eur J Med Res 2018;23:58.

8. Mor JM, Guo Y,Koch KR ,Heindl LM. Transcanalicular Diode Laser-Assisted Dacryocystorhinostomy for the Treatment of Primary Acquired Nasolacrimal Duct Obstruction. Department of 
Ophtalmology University of Cologne J Vis Exp, 2017;128: 55981.

9. Raposo A, García-Purriños F, Piqueras F, Martínez-Martínez M, Lajara J. Anatomical Variants and Bilateral Lacrimal Pathways Surgery: Avoiding Unnecessary Surgery. Eur Arch Otorhinolaryngol. 2017;274:3135-8.

10. Raposo A, Piqueras F, García-Purriños F, Martínez-Martinez ML, Lajara J. Influence of Septal Deviation on the Prognosis of Transcanalicular Diode Laser-Assisted Dacryocystorhinostomy. J Ophthalmol. 2016;2016:9573760.

11. Yener HI, Gul A, Caglar C, Ozcimen M. The Effectiveness of Postoperative Early Ostium Clea- ning in Transcanalicular Diode Laser-Assisted Dacryocystorhinostomy. Ophtahalmic Plast Reconstr Surg 2014; 30(6):476-9.

12. Rosique López L, Lajara Blesa J, Rosique Arias M. Utilidad de las Curas tras Dacriocistorrinostomia Láser. Acta Otorrinolaringol Esp. 2013; 64(4):279-282.

13. Dolmetsch AM. Nonlaser Endoscopic Endonasal Dacryocystorhinostomy with Adjunctive Mitomicyn C in Nasolacrimal Duct Obstruction in Adults. Ophthalmology. 2010;117(5):1037-40.

14. Gupta N. Improving Results in Endoscopic DCR. Indian J Otolaryngol Head and Neck Surg 2011;63(1):40-4. 